\section{Optimizing the Sampling Design of Morphometric Experiments}

\author{
John M. Basgen \\ University of Minnesota \\ basgen@umn.edu
}

In a previous article [1], stereological methods for measuring volume, surface, length, and number were described. The present article will briefly discuss sampling methods, as well as techniques for optimizing the number of animals per group and the number of measurements per animal when planning a morphometric study.

\section{SAMPLING:}

In many biological studies, we are interested in finding the average measure of some structural parameter in a population of individuals, and perhaps to determine whether a certain treatment affects this average measure. It usually is not possible to measure all the individuals belonging to a population, therefore, some selection or sampling of the population must be performed. But, when only a sample is measured, it is not possible to know the true average of the population, and only an estimate of "The Truth" is possible. For this estimate to be useful, it must be accurate (unbiased). It is not possible to obtain an unbiased estimate from a biased sample. Alan Stuart states in his book "Basic Ideas of Scientific Sampling", "...we cannot allow ourselves to be guided in our sampling choice by mere convenience, speed, cheapness, or the lack of an obvious reason against what we are doing. In sampling it is never enough not to have detected a bias - we must ensure by our sampling methods that no possibility of a bias can arise" [2]. Unfortunately, it is not possible to know if a sample is free from bias by looking at the data obtained from the sample. In fact, unless you know "The Truth" a priori, you cannot know if the data is biased or not. And, of course, if one knew "The Truth" a priori, one would not bother to do the experiment. Therefore, since one cannot know if the sample is free of bias by looking at the data, much effort should be placed in designing a sampling scheme that does not allow bias in the first place. The following list discusses several possible sampling designs.

1. Arbitrary sampling requires that little or no thought be given to the design process. An example of arbitrary sampling is to place rats in different groups by assigning the first half of the animals taken from their shipping box to experimental group I, and the remaining animals to group II. This arbitrary method may result in the timid or weaker animals being grabbed first and assigned to Group I. A second example of arbitrary sampling is related to the selection of tissue blocks. If one region of an organ is cut into blocks and a few are chosen arbitrarily for embedment, this region would be over represented while other areas would be under represented. Thus, convenient arbitrary designs may result in biased samples. Some sampling designs described as random in journal articles are probably, in fact, arbitrary.

2. Random sampling assures that every individual in a population or all potential blocks from an organ have an equal chance of being selected for analysis. Obtaining a random sample is not a trivial task. Even if we assume that the rats we receive from one vendor are a random sample of the population, how do we randomly assign each rat to an experimental group? We could arbitrarily number the rats from 1 to $\mathrm{n}$, and then use a random number generator to assign animals to each experimental group. This would work with rats, but what about blocks? A grid of numbered rows and columns can be superimposed over the organ, and then a random number generator used to choose the positions from where each block is selected. It turns out true random samples are free from bias but are inefficient [3].

3. Systematic sampling produces unbiased and efficient samples. Animals can be arranged in some logical order to produce a systematic sample. For example, if the parameter to be measured is related to body size, the animals can be arranged according to weight. And then moving from smallest to largest, the animals can be alternately assigned to the different experimental groups. Use a random number to determine which group receives the first animal. The fractionator technique [4] very efficiently produces systematic, unbiased tissue samples. When using this technique, one cuts an organ into slices, systematically selects a fraction of the slices, and cuts them into strips. Next, a fraction of the strips is systematically selected and cut into blocks, and finally a fraction of the blocks is systematically selected and embedded for sectioning. The fractionator technique results in a true systematic and unbiased sample of the entire organ.

\section{HOW MANY TO MEASURE?}

When planning a morphometric experiment, the questions "How many animals per group?", "How many blocks per animal?", and "How many measurments per block?" should be considered. These are complex but answerable questions. The answers depend on factors such as: 1) Variability of the specific parameter among animals within the group (inter-animal variability); 2) Variability of the specific parameter within each animal, including measuring error (intra-animal variability); and 3) magnitude of the difference between group means. If all the animals in a group were the same, only one animal would have to be measured. If all blocks from an animal were the same, only one block would need to be studied, and if all measurments from a block were the same, only one measurement per block would need to be made. Rarely does this happen; thus, more than one animal per group, more than one block per animal, and more than one measurement per block must be utilized. Using too many animals or too many blocks per animal, or making too many measurments per block wastes time and money. Therefore, it is reasonable to make some effort to optimize the sampling design at the beginning of each new experiment.

The method for determining the number of animals per group depends on the magnitude of the difference between group means, the magnitude of group variances, the significance level ( $\alpha$ ), and the level of power (1- $\beta$ ) needed for the particular experiment. This method is described in many statistical books [5] and will not be presented here.

The technique referred to as "partitioning the variance" can be used to optimize the number of blocks per animal and the number of measurments per block $[6,7,8,9]$. This method determines the relative variability at the different sampling levels and thus can be used to determine the optimal sample size at the different sampling levels. The data necessary to calculate the relative amount of interanimal and intra-animal variability sometimes can be obtained from the literature, but more likely a pilot study will be needed. A pilot study often consists of about 3-5 animals in a group, 2-3 blocks per animal, and 5-10 measurements per block.

To demonstrate the calculations necessary to partition the variance, data from a simple non-microscopy study was used. This example determines the number of times each rat needs to be weighed in order to find the average body weight of the group. Five rats were weighed. As each rat was weighed, there was an uncertainty to the measure (i.e., the rats move around during weighing, thus making it difficult to read the scale precisely). Therefore, 
$\checkmark \quad$ Lehigh Microscopy Schools

June 8, Introduction to SEM \& EDS

June 9-13, SEM \& X-ray Microanalysis

June 16-20, Advanced SEM

June 16-20, Quantitative X-ray Microanalysis

June 16-19, Analytical TEM

June 16-19, Characterization of Nanostructures

June 16-19, Particle Characterization

June 17-19, TEM Specimen Preparation

June 17-20 Atomic Force Microscopy

sharon.coe@lehigh.edu

$\checkmark$ SCANDEM 2003, Scandinavian Soc. for Microscopy

June 10-13, 2003, Oslo, Norway

www.scandem2003.uio.no

$\checkmark \quad$ FEMS 2003, Congress of European Microbiologists

June 29-July 3, 2003, Ljubljana, Slovenia

www.fems-microbiology.org

$\checkmark \quad$ Microscopy and Microanalysis 2003

August 3-7, 2003, San Antonio, TX

www.msa.microscopy.com

$\checkmark$ EUROMAT 2003 European Cong. Adv. Mat. \& Processes September 1-5, 2003, Lausanne, Switzerland www.euromat2003.fems.org

$\checkmark \quad$ Electron Microscopy and Analysis Conferences 2003 September 3-5, 2003, Oxford, UK www.physics.iop.org/IOP/Confs/EMG

$\checkmark$ MC 2003 Microsc. Conf.-International Forum Adv. Micr. September 7-12, 2003, Dresden, Germany www.mc2003.tu-drresden.de

$\checkmark \quad$ BCEIA 2003 Beijing Conf. Instrumental Analysis October 14-17, 2003, Beijing, China www.instrument.com.cn/bceia/

$\checkmark$ National Society for Histotechnology

October 18-23, 2003, Louisville, KY

histo@nsh.org

$\checkmark \quad$ 8th Annual RMC Materials Microtomy Course

October 28-31, 2003, Tucson, AZ

www.rmcproducts.com

$\checkmark$ Eastern Analytical Symposium

November 2-8, 2003, Santa Clara, CA

www.eas.org/news.htnl

$\checkmark \quad$ Society for Neuroscience

November 8-13, 2003, New Orleans, LA

web.sfn.org

$\checkmark \quad$ American Society for Cell Biology 2003

December 13-17, 2003, San Francisco, CA

www.ascb.org

$\checkmark \quad$ Materials Research Society

December 1-5, 2003, Boston, MA

info@mrs.org

$\checkmark \quad$ Microscopy and Microanalysis 2004

August 1-5, 2004, Savannah, GA

www.msa.microscopy.com

$\checkmark \quad$ EMC 2004 (former EUREM)

August 22-27, 2004, Antwerp, Belgium

www.emc2004.be

$\checkmark \quad$ Microscopy and Microanalysis 2005 July 31-August 4, 2005, Honolulu, HA www.msa.microscopy.com

Please check the "Calendar of Meetings and Courses" in the MSA journal "Microscopy and Microanalysis" for more details and a much larger listing of meetings and courses.
The objective of this publication is simply to provide material of interest and value to working microscopists!

The publication is owned by the Microscopy Society ofAmerica (MSA) and is produced six times each year in odd months, alternating with MSA's peer-reviewed, scientific journal Microscopy and Microanalysis. We greatly appreciate article and material contributions from our readers- "users" as well as manufacturers/suppliers. The only criteria is that the subject matter be of interest to a reasonable number of working microscopists. Microscopy Today has authors from many disparate fields in both biological and materials sciences, each field with it's own standards. Therefore $M T$ does not have a rigid set of style instructions and encourages authors to use their own style, asking only that the writing be clear, informative, and accurate. Length: typical article length is 1,500 to 2,000 words plus images, Longer articles will be considered,

Short notes are encouraged for our Microscopy 101 section.

\section{MICROSCOPY TODAY}

\author{
Ron Anderson, Editor \\ ron.anderson@attglobal.net \\ José Mascorro, Technical Editor \\ jmascor@tulane.edu \\ Dale Anderson, Art Director \\ dale.anderson@attglobal.net \\ Regular Mail to: \\ PO Box 499 \\ Wappingers Falls, NY 12590 \\ Courier Mail to: \\ 21 Westview Drive \\ Poughkeepsie, NY 12603 \\ Telephones: \\ 1-(845)463-4124 - Fax: (845)463-4125 \\ e-Mail: \\ microtoday@attglobal.net \\ WWW Page: \\ http://www.microscopy-today.com
}

Total Circulation: 15,741 (4/30/2003)

Disclaimer: By submitting a manuscript to Microscopy Today, the author warrants that the article is original (or that the author has the right to use any material copyrighted by others). The use of trade names, trademarks, etc., does not imply that these names lack protection by relevant laws and regulations. Microscopy Today, the Microscopy Society of America, and any other societies stated, cannot be held responsible for opinions, errors, or for any consequences arising from the use of information contained in Microscopy Today. The appearance of advertising in Microscopy Today does not constitute an endorsement or approval by the Microscopy Society of America of the quality or value of the products advertised or any of the claims, data, conclusions, recommendations, procedures, results or any information found in the advertisements. While the contents of this magazine are believed to be accurate at press time, neither the Microscopy Society of America, the editors, nor the authors can accept legal responsibility for errors or omissions.

(C) Copyright, 2003, The Microscopy Society of America. All rights reserved. 


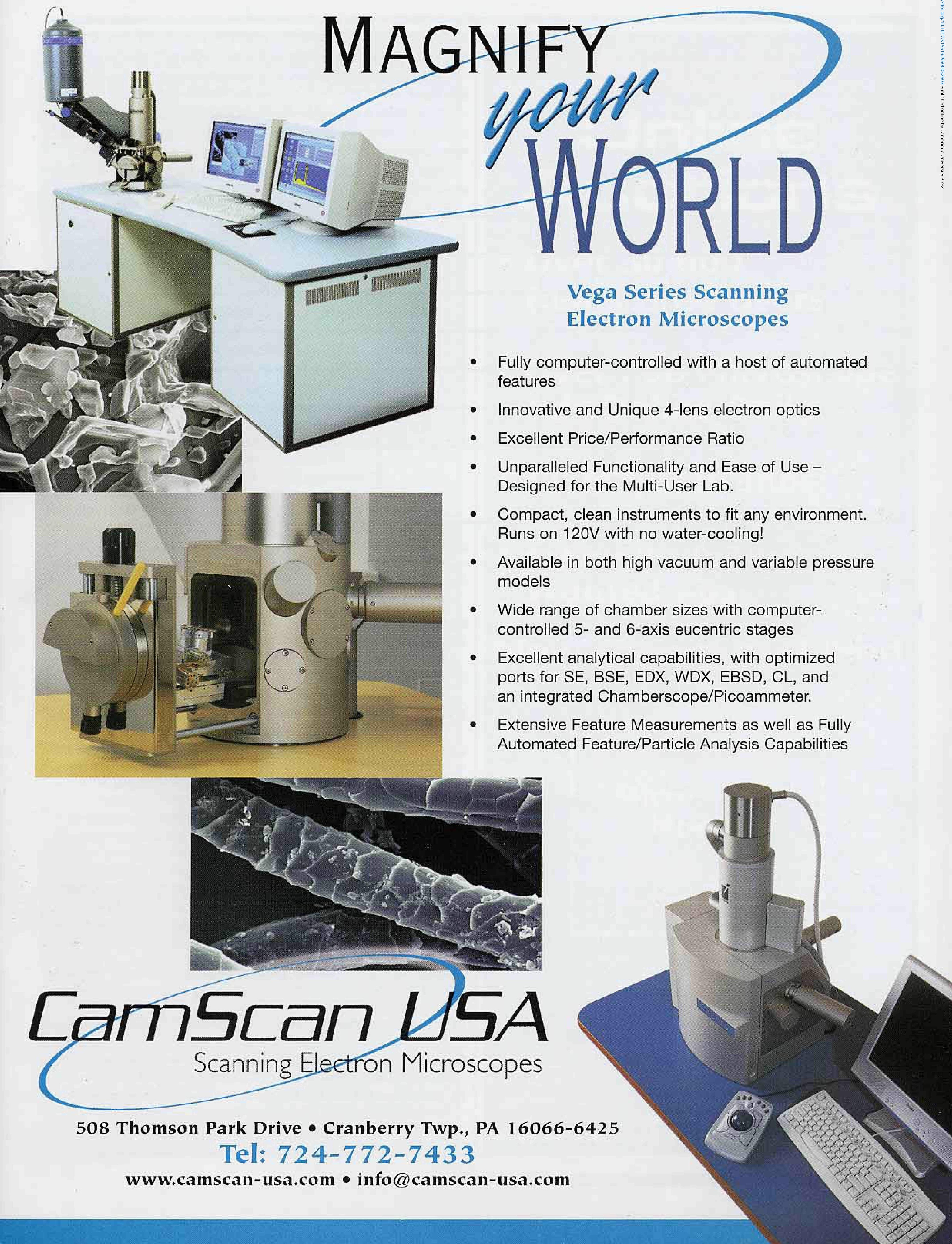


Continued from page 5

each rat was weighed twice. After weighing, the mean standard deviation (SD) and standard error of the mean (SEM) were calculated for each animal. Next, the observed coefficient of error (OCE=SEM/mean) and $O C^{2}$ were calculated for each animal. Finally the group mean, group SD, group observed coefficient of variation ( $\mathrm{OCV}=$ Group $\mathrm{SD} / \mathrm{Group}$ mean), and mean group $\mathrm{OCE}^{2}$ were calculated (Table 1).

Because the coefficient of error and coefficient of variation are

measurments) and therefore it would be a waste of time to weigh each animal more than once in the subsequent study.

A rule to determine if more or less measurments should be use is to have the $O \mathrm{OE}^{2} \leq 1 / 2 \mathrm{CV}^{2}$. The Gundersen paper "Do more less well!" [6] offers a good introduction to partitioning of variance. The book by Howard and Reed, Unbiased Stereology, [9] also includes a good explanation concerning this topic.

Because biological variation usually overwhelms the intraanimal variations (unless the measuring technique is poor), it is

\begin{tabular}{|c|c|c|c|c|c|c|c|}
\hline Rat \# & Weight 1 & Weight 2 & Mean & $S D$ & SEM & OCE & $O C E^{2}$ \\
\hline 1 & 307 & 309 & 308 & 1 & 0.7 & 0.002 & 0.000004 \\
\hline 2 & 305 & 307 & 306 & 1 & 0.7 & 0.002 & 0.000004 \\
\hline 3 & 302 & 304 & 303 & 1 & 0.7 & 0.002 & 0.000004 \\
\hline 4 & 305 & 303 & 304 & 1 & 0.7 & 0.002 & 0.000004 \\
\hline 5 & 306 & 306 & 306 & 0 & 0.0 & 0.000 & 0.000000 \\
\hline & & \multicolumn{2}{|c|}{$\begin{array}{l}\text { Group Mean: } 305 \\
\text { Group SD: } 2 \\
\text { Group OCV: } 0.0066\end{array}$} & & & \multicolumn{2}{|c|}{$\begin{array}{c}\text { Mean Group OCE } 2 \\
0.000003\end{array}$} \\
\hline
\end{tabular}
not necessary to make thousands of measurments per animal as was believed necessary in the past [11]. Usually only 100-200 measurements per animal are sufficient [12]; however, the measurements should be distributed wisely and without bias throughout the tissue of interest.

Using modern stereological and

calculated from observed data, they are referred to as observed coefficient of error and observed coefficient of variation. The relationship between these measures is given by the equation:

$$
\underset{\text { Equation } 1}{O C V^{2}} \mathrm{OCE}^{2}
$$

where $\mathrm{CV}$ is the inherent biological variation among the animals within the group. To determine this biological variation, in equation 1 we replace $\mathrm{OCV}$ with 0.0066 and $\mathrm{OCE}^{2}$ with 0.000003 and solve for $\mathrm{CV}^{2}$.

$$
\begin{aligned}
& (0.0066)^{2}=C V^{2}+0.000003 \\
& \text { Equation 2 }
\end{aligned}
$$

Thus, $C V^{2}$ equals 0.000041 which leads to Equation 3 .

$$
\begin{aligned}
& 0.000044=0.000041+0.000003 \\
& \text { Equation } 3
\end{aligned}
$$

Finally, set the $O C V^{2}$ equal to $100 \%$ of the variation and calculate the percent variation contributed by $\mathrm{CV}^{2}$ and $O C E^{2}$.

$$
\begin{aligned}
& 100 \%=0.000041 / 0.000044+0.00003 / 0.000044 \\
& \text { Equation } 4
\end{aligned}
$$

So, $93 \%$ of the observed variation is due to biological variation among the animals and only $7 \%$ is intra-animal variation or measuring error. Biological variation is determined by "Mother Nature" and can not be changed by the experimenter. The experimenter, however, can change the intra-animal variation by changing the sample size [10]. Increasing the number of measurements per animal will decrease the percentage of variation contributed by the measuring error. In this pilot study, the biological variation greatly overwhelms the intra-animal variation (imprecision of the weight sampling techniques allows for precise and accurate measurement of volumes, areas, lengths, and number in a very efficient manner.

\section{REFERENCES}

1. Basgen JM. (2002) Basic stereology. Microscopy Today 11 (1) 12-16.

2. Stuart A. (1976) Basic Ideas of Scientific Sampling, Second Edition, Charles Griffin \& Co., London, pages 10-11.

3. Weibel ER. (1979) Stereological Methods Practical Methods for Biological Morphometry, Academic Press, New York, pages 82-83.

4. Gundersen HJG. (1986) Stereology of arbitrary particles. Journal of Microscopy 143:3-45.

5. Rosner B. (1982) Fundamental of Biostatistics, Duxbury Press Boston pp.189-194.

6. Gundersen HJG and Østerby R. (1981) Optimizing sampling efficiency of stereological studies in biology: or "Do more less well!". Journal of Microscopy 121:65-73.

7. Gupta M, Mayhew TM, et al. (1983) Inter-animal variation and its influence on the overall precision of morphometric estimates based on nested sampling design. Journal of Microscopy 131:147-154.

8. Mayhew TM. (1983) Stereology: progress in quantitative microscopical anatomy. Progress in Anatomy Volume 3. Editors: V Navaratnam and RJ Harrison pp. 81-112.

9. Howard CV and Reed MG. (1998) Unbiased Stereology: Three-Dimensional Measurement in Microscopy, Bios-Springer Publishing, New York, pp.163-165.

10. Cruz-Orive L. (1994) Toward a more objective biology. Neurobiology of Aging 15:377-378.

11. Weibel ER. (1979) Stereological Methods Practical Methods for Biological Morphometry, Academic Press, New York, pp. 110-116.

12. Cruz-Orive LM, Weibel ER (1990) Recent stereological methods for cell biology: a brief survey. American Journal of Physiology 258(4pt1):L148-156. 


\section{LEO SUPRA...}

\section{The ultra-high resolution, variable pressure analytical FESEM}

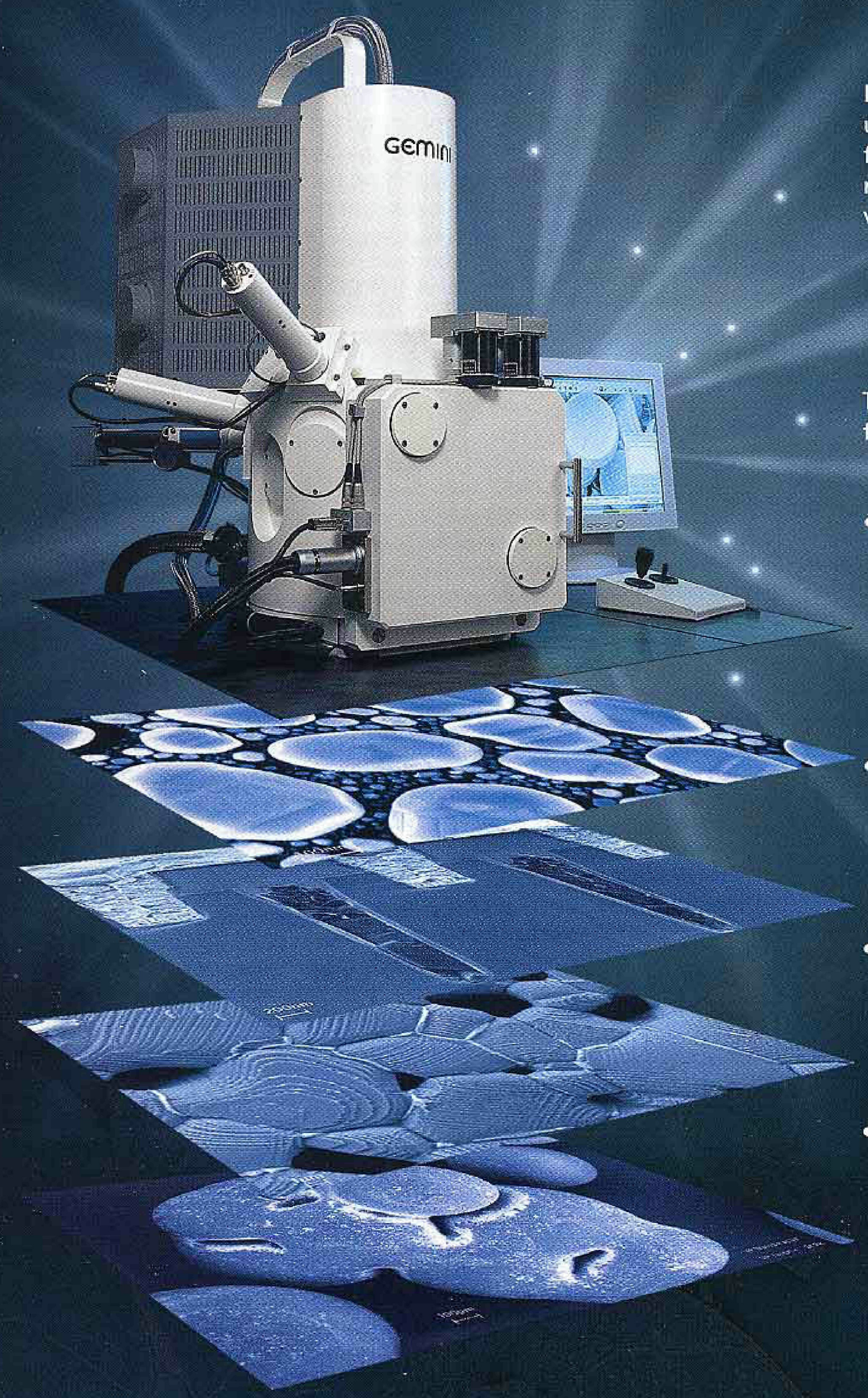

LEO SUPRA is the versatile, ultra-high resolution FESEM for solutions to semiconductor. materials, life science and variable pressure applications.

The new LEO SUPRA combines four instruments into one:

q. The LEO SUPRA delivers ultra-high resolution over the complete voltage range: $1.0 \mathrm{~nm} @ 15 \mathrm{kV}$

$1.7 \mathrm{~nm} @ 1 \mathrm{kV}$ $4.0 \mathrm{~nm} @ 0.1 \mathrm{kV}$

- Large sample chamber and fully motorized precision stage handles oversized specimens

- Full analytical FESEM with probe currents up to $20 \mathrm{nA}$ for EBSD and WDX applications

- LEO's proprietary variable pressure technology allows investigation of non-conducting specimens by eliminating charging without prior sample preparation

LEO Electron Microscopy Group A Carl Zeiss SMT AG Company 73417 Oberkochen, Germany Tel: $++4973 \quad 64-946137$ Fax: $++4973 \quad 64-944851$ info@leo.de • www.leo-em.com
LEO Electron Microscopy Ine A Carl Zeiss SMT AG Company Thornwood, New York 10594 Toll Free: $800-356-1090$ Fax: $914-681-7443$ www.leo-usa.com

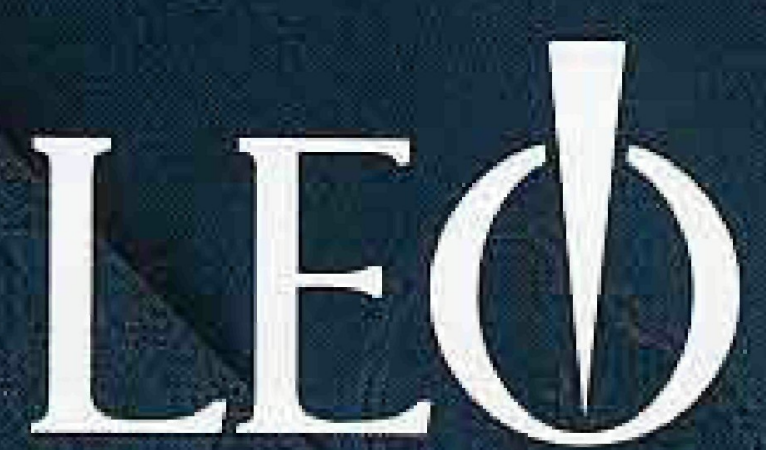

